\title{
FE Analysis on Contact Properties between Root- Blade and Slot-Disc Boping Wang ${ }^{1, a}$,Jiao Wang ${ }^{2, b}$ Xingzhan Li ${ }^{2, c}$, Qingkai Han* 2,d \\ ${ }^{1}$ Department of Flight Theory, Aviation University of Air Force,Changchun,130022,China \\ ${ }^{2}$ School of Mechanical Engineering, Northeastern University, Shenyang,110819, China

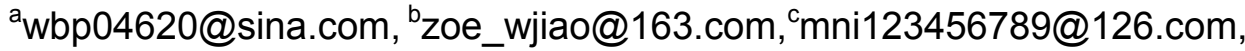 \\ *dqhan@mail.neu.edu.cn (Corresponding author)
}

\section{Keywords: Contact properties; Root-Blade; Slot-Disk; Different rotating speeds}

\begin{abstract}
The contact problem between root-blade and slot-disk structure of compressor is of much importance, because there is serious stress concentration in the structure, which is one of the parts with multi faults in aero-engine. First, finite element model for dovetail attachment is established to analyze stress concentration effects. Then, the stress distribution and other parameters such as contact pressure is obtained through contact analysis. In this paper, the finite element method based on contact analysis is used. Calculating results show that equivalent stress, contact pressure of blade-disk structure increase steadily as the rotating speed increases.
\end{abstract}

\section{Introduction}

Contact phenomenon is involved in many descriptions of engineering problems, taking aero-engine for example, including interference fit when assembled and blade-disc dovetail joint structure. Interaction of contact objects in the contact interface is more complex, which is the main reason for occurrence of damage and destruction. Aero-engine blade-disc dovetail joints are typical contact structures. At the edge of contact regions fretting can occur under thermo-mechanical loads. This fretting can lead to fatigue crack initiation and to ultimately failure of the dovetail joint structure [1]. With the growing structure life, contact fatigue and fretting fatigue are emphasized increasingly in engineering $[2,3]$.

In recent years, some research with engineering significance for contact analysis of blade-disc dovetail joint structure is carried out. An early contribution is Boddington et al[4], which considers Two-dimensional elastic analysis. This paper explores the feasibility of implementing Amonton's law with finite elements and is successful in devising an approach for doing this. Amonton's law ( $F=\mu N$ ) is typical dry friction law, which says that the frictional force $F$ is linearly proportional to load $N$, and does not depend on apparent contact area of two solid surfaces and sliding velocity. Two-dimensional elastic finite element analysis is Kenny et al. In order to validate the results of two-dimensional finite element analysis, photoelastic tests were conducted in this paper [5].Other papers which research two-dimensional elastic finite element analysis are Papanikos and Meguid[6], and Meguid et al[7]. Three-dimensional elastic finite element analysis of dovetail joints consider the effect of critical geometrical features, such as flank length, flank angle, fillet radii ,skew angle and friction coefficients upon the resulting stress field; however, it is observed that the level of mesh refinement is not adequate to capture stress convergence[8]. To solve this issue, sub-modeling approach has been implemented in 3D analysis to obtain converged contact stress results for the a straight dovetail with flat interface [9].Other papers which refine grids with mesh sizes near the edges of contact to improve the solving accuracy of contact stress are Sinclair $G$ $\mathrm{B}$ et al [1] , and Wei et al [10]. Finite element method is applied to analyze contact stress of dovetail joint. Several geometries are chosen to study the effect of critical geometrical parameters on stress 
distribution, such as flank angle, flank length, fillet radii at the edge of contact region and profile of contact surface. The numerical research focuses on an arc/line contact profile in 2D and discusses the geometrical parameters of contact surface to improve contact stress distribution of contact region. Results indicated that reasonable geometrical parameters can improve contact stress distribution of contact region to increase the structure life [11]. Three-dimensional loading effect is brought out by considering a skewed dovetail slot in the geometry and by application of forces and moments acting on the airfoil. The study suggests that skew angle and three-dimensional loading need to be considered, for an improved understanding of the fretting aspects of a dovetail interface [12]. The above review shows that existing research in blade-disc dovetail joints contact analysis has been primarily concerned with effect of contact parameters varied on stress distribution of contact region. However, less research is conducted by considering the effects of rotating speeds of blade-disk structure on contact parameters.

This paper considers the effects of rotating speeds on contact parameters such as equivalent stress and contact pressure.

\section{Finite element modeling}

Finite element mesh generation of the bladed-disc rotor modeling is based on finite element software ANSYS ICEM. Figure 1 shows the details of global finite element model of rotor sector with straight dovetail. The material used for the modeling of blade-disc is Titanium Alloy Ti-6Al-4V with Young's modulus $E=89 \mathrm{Gpa}$, Poisson's ratio $v=0.3$, and density $\rho=4.42 \mathrm{~g} / \mathrm{cm}^{3}$.

The 3D finite element model is built using eight node solid element of Solid185.

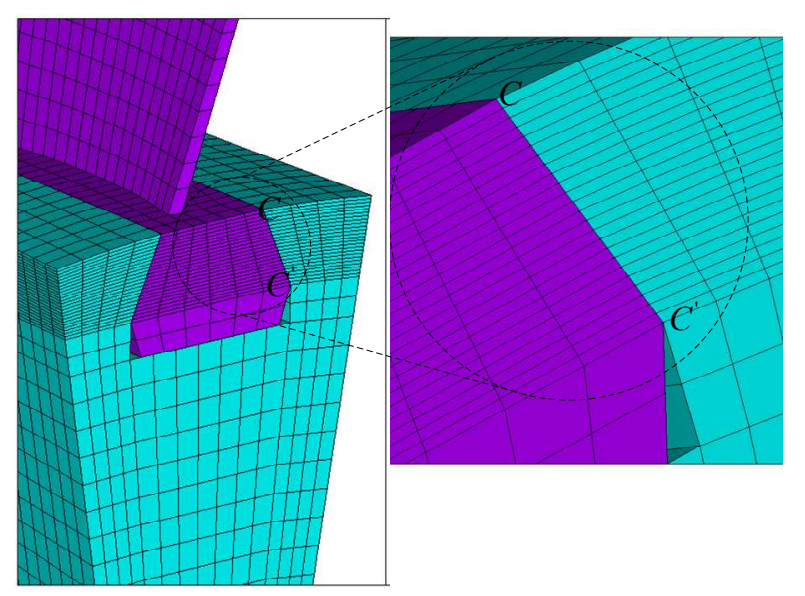

Fig. 1 FE modeling of Bladed-disc rotor

Disc and shaft connection areas are the fixed boundary conditions. Load conditions are the centrifugal forces of the blade and disc as a result of rotation, and contact stress between root-blade and slot-disc under the centrifugal forces. The maximum rotating speed of blade-disc is set to $8800 \mathrm{r} / \mathrm{min}$, and considering different rotating speeds have an effect on contact analysis.

\section{The effect of rotating speeds on contact parameters}

Contact analysis is done by using finite element software ANSYS. Blade-disc structure analyzed is subjected to centrifugal loading only with a constant angular velocity. Considering respectively effects of $3000 \mathrm{r} / \mathrm{min}, 4500 \mathrm{r} / \mathrm{min}, 6000 \mathrm{r} / \mathrm{min}, 7500 \mathrm{r} / \mathrm{min}$ and $8800 \mathrm{r} / \mathrm{min}$ on contact parameters, including friction coefficient is set to 0.4 , contact stiffness is set to 0.01 in ANSYS. 
Relations between equivalent stress and rotating speeds. Figure 2 reveals that equivalent stress of nodes at the edge of contact and blade-disc structure gradually increase as the rotating speeds increases.

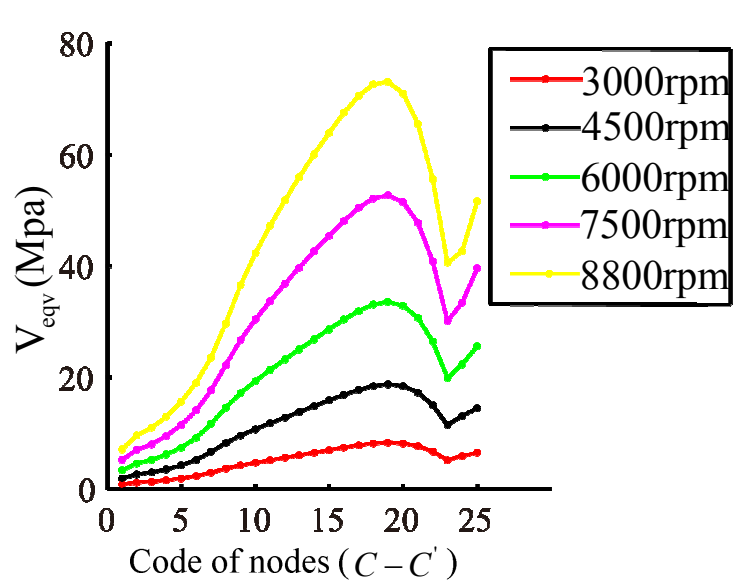

(1) Relations between equivalent stress of nodes at the edges of contact and rotating speeds

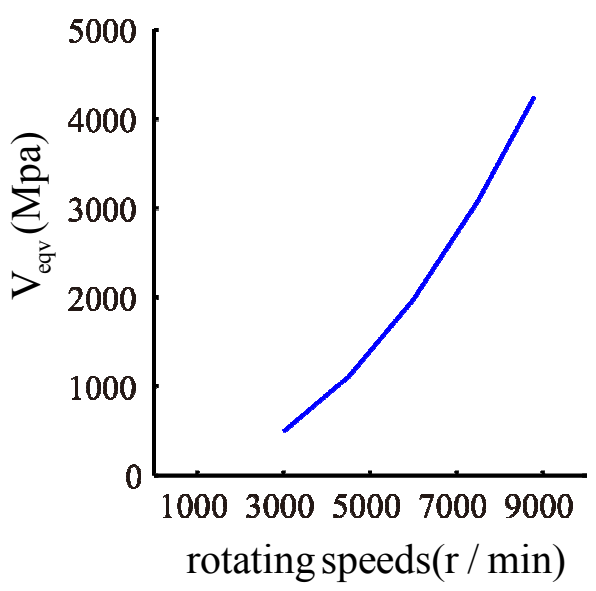

(2) Relations between equivalent stress of the blade-disc modeling and rotating speeds

Fig. 2 Curve of von equivalent stress vs. rotating speeds

Relations between contact pressure and rotating speeds. Figure 3 shows that contact pressure of nodes at the edges of contact and blade-disc structure increase with rotating speeds increasing, and the higher the rotating speeds, the greater the contact pressure difference.

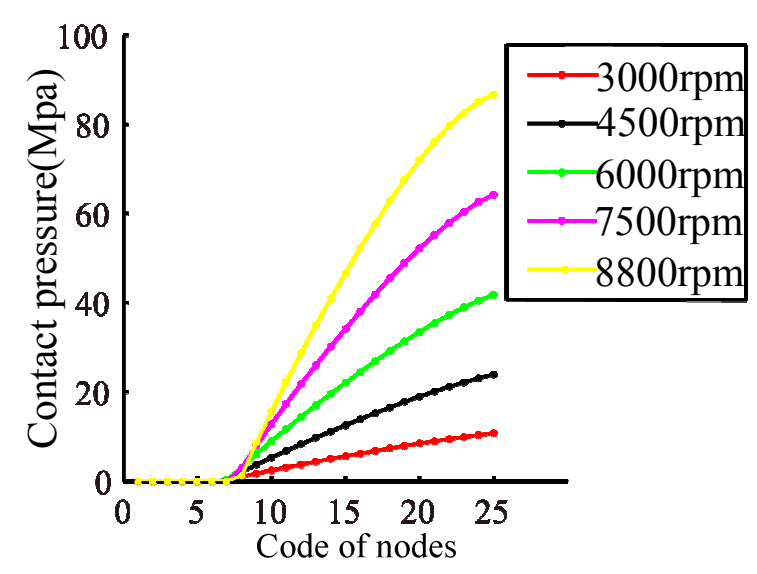

(1) Relations between contact pressure of nodes at the edges of contact and rotating speeds

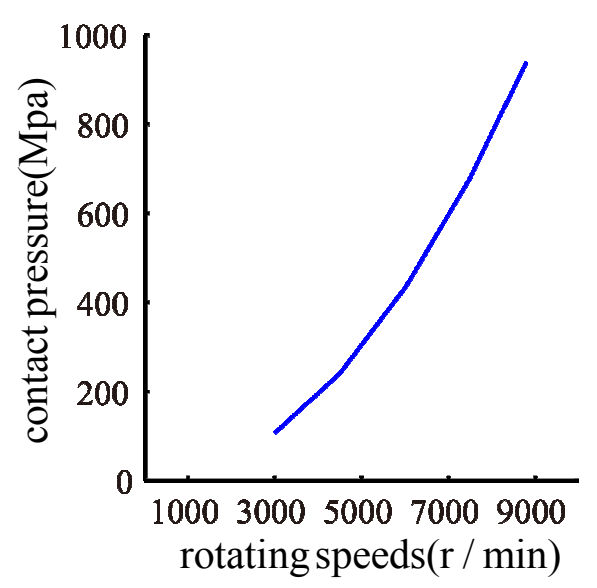

(2) Relations between contact pressure of the blade-disc modeling and rotating speeds

Fig. 3 Curve of contact pressure vs. rotating speeds

\section{Conclusions}

In this paper, contact analysis of root-blade and slot-disc is conducted, considering the effect of different rotating speeds on the contact parameters. The contact analysis of root-blade and slot-disc at different rotating speeds concludes that contact pressure and equivalent stress of the whole modeling, and contact pressure, equivalent stress of nodes at the edges of contact gradually increase as the rotating speed increases. 


\section{Acknowledgement}

The work was supported by the Fundamental Research Funds for the Central Universities (Grant No. N090603007) and Natural Science Foundation of China (Grant No. 51175070).

\section{References}

[1] Sinclair G B, Cormier N G, Griffin J H, et al, Contact stresses in dovetail attachments: finite element modeling, J. Journal of Engineering for Gas Turbines and Power. 124 (2002) 182-189.

[2] Gu, Y.X., Research on fretting fatigue life of dovetail joint under HCF-LCF Load, Nanjing: Nanjing University of Aeronautics and Astronautics, 2007.

[3] Liu D.X., J. Liu, Y.Y. Liu, Study on nucleating location and formation of fretting fatigue cracks, J. Engineering mechanics. 24 (2002) 42-47.

[4] Boddington, P.H. B., Chen, K., Ruiz, C., The numerical analysis of dovetail joints, J. Comput. Struct. 20 (1985) 731-735.

[5] Kenny, B., Patterson, E. A., Said, M., Aradhya, K. S. S., Contact Stress Distributions in a Turbine Disk Dovetail Type Joint-A Comparison of Photo elastic and Finite Element Results, J. Strain. 27 (1991) 21-24.

[6] Papanikos, P., and Meguid, S. A., Theoretical and experimental studies of fretting-initiated fatigue failure of aero engine compressor discs, J. Fatigue Fract. Eng. Mater. Struct. 17 (1994) 539-550.

[7] Meguid, S. A., Refaat, M. H., and Papanikos, P., Theoretical and experimental studies of structural integrity of dovetail joints in aero engine discs, J. Mater. Process. Technol. 56 (1996) 668-677.

[8] Papanikos, P., Meguid, S. A., and Stjepanovic, Z., Three-Dimensional Nonlinear Finite Element Analysis of Dovetail Joints in Aero engine Discs, J. Finite Elem. Anal. Design. 29 (1998) 173-186.

[9] Beisheim J. R., Sinclair G. B., On the three-dimensional finite element analysis of dovetail attachments, J. ASME Journal of Turbo machinery. 125 (2003) 372-379.

[10] Wei, D.S., Wang Y.R., Effects of profile of contact surfaces on the stress distribution for tenon jointing in blade disk assemblies, J. Journal of Aerospace Power. 25 (2010) 407-411.

[11] Wei, D.S., Wang Y.R., Effects of critical geometrical parameters on distribution of contact stress in a tenon jointing, J. Journal of Propulsion Technology. 4 (2010) 473-477.

[12] Anandavel K., Prakash, R. V., Effect of three-dimensional loading on macroscopic fretting aspects of an aero-engine blade-disc dovetail interface, J. Tribology International. 10 (2010) $1-12$. 